\title{
CORTICAL GROWTH IS IMPAIRED BY VARIABLE HYPEROXIA IN A PRETERM MODEL OF GROWTH RESTRICTION
}

\author{
E. Woodrow ${ }^{1}$, P. Georgala ${ }^{2}$, J. Wade $^{1}$, J. Becher $^{1}$
}

${ }^{1}$ Jennifer Brown Research Laboratory, MRC Centre for Reproductive Health, Queens Medical Research Institute, University of Edinburgh, ${ }^{2}$ Genes and Development Group, Centres for Integrative Physiology and Neuroscience Research, University of Edinburgh, Edinburgh, UK

Background and aims: Chronic lung disease of prematurity is strongly associated with adverse neurodevelopmental outcome. Oxygen therapy has been implicated as a factor leading to preterm brain injury. Preterm infants with intrauterine growth restriction (IUGR) are at increased risk of both chronic respiratory morbidity and neurodevelopmental impairment. We determined the effect of postnatal variable hyperoxia on the motor cortex in a rodent model of IUGR.

Methods: Sprague-Dawley dams were fed $18 \%$ or $9 \%$ protein diet from E15 to P7. Pups were reared in air or variable hyperoxia from birth until P7. Brains were weighed and sections through the motor cortex were stained with cresyl violet. The thickness of the superficial (II-IV) and deep (V-VI) layers of the motor cortex was measured using ImageJ analysis. Staining for neuronal subtypes was performed using immunofluorescence and cell numbers were quantified.

Results: Protein restricted pups had smaller brains than normally grown pups at P7 ( $<<0.0001)$ but cortical thickness was preserved. Postnatal variable hyperoxia did not further affect brain weight at P7 in either group but did result in a decrease in cortical thickness in growth restricted pups $(p=0.003)$. Thinning was observed in both superficial $(\mathrm{p}=0.01)$ and deep layers $(\mathrm{p}=0.03)$. However total numbers of superficial, callosal, corticothalamic and corticospinal neurons were preserved.

Conclusions: Postnatal variable hyperoxia, a modifiable factor in neonatal care, impairs cortical growth without reducing total brain weight or neuron numbers in a preterm model of IUGR. This implies that the reduction in cortical thickness may be secondary to impaired cortical circuitry. 